\title{
2013: un anno molto intenso ... e siamo già pronti per il 2014!
}

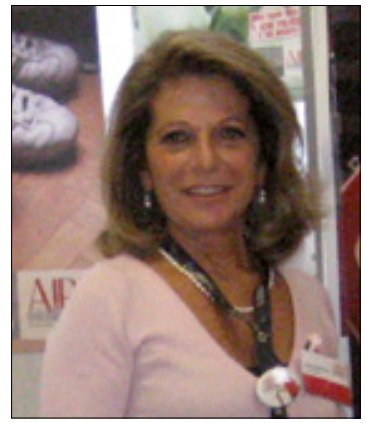

\section{Luisa Sternfeld Pavia}

Presidente

Associazione Italiana Rene

Policistico AIRP onlus

luisa.sternfeld.airp@renepolicistico.it
Cari lettori, cari amici,

l'anno volge ormai al termine. Il 2013 ha segnato momenti davvero importanti per la nostra Associazione, pieni di grandi soddisfazioni e caratterizzati da un'intensa attività.

Purtroppo, oltre ai bei momenti, il 25 Ottobre scorso abbiamo avuto il dispiacere di dover affrontare una grande e improvvisa perdita: a Maria Giovanna Flaùto, Responsabile della Sezione AIRP di Ancona, vanno tutti i nostri pensieri.

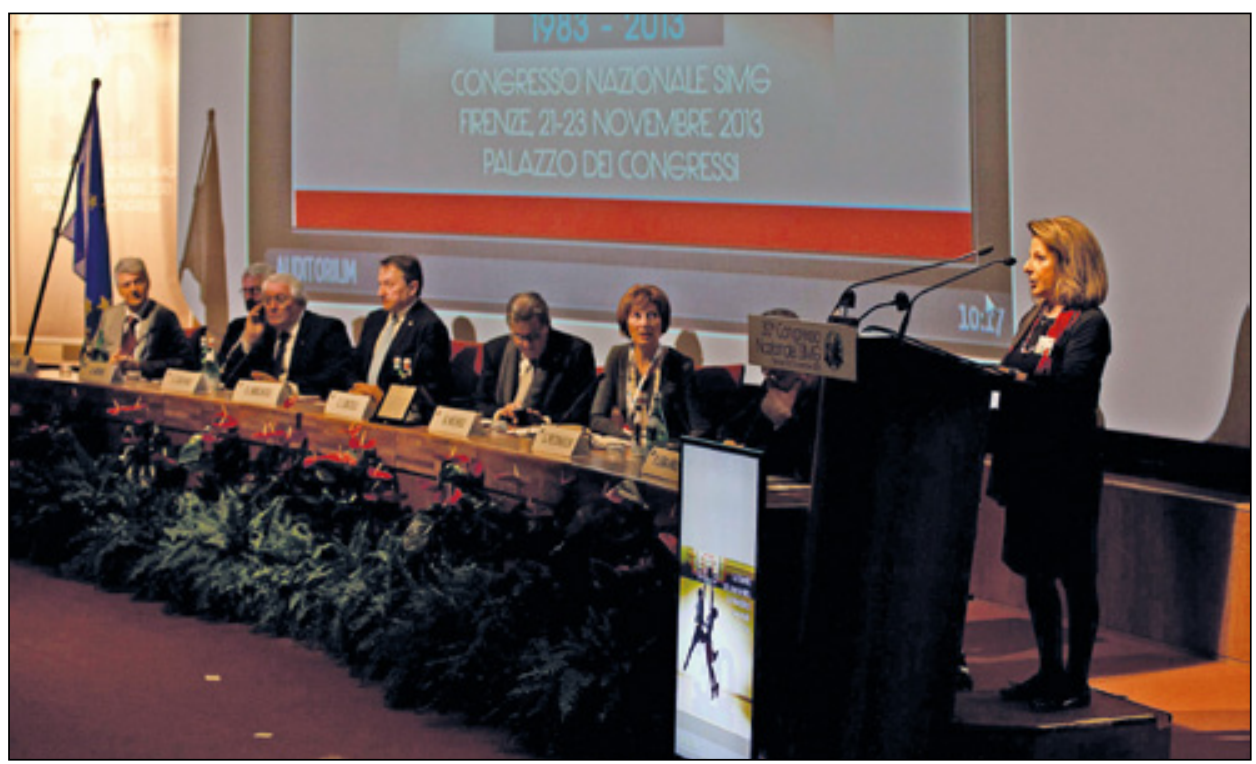

In questo ultimo numero dell'anno, vorrei ricordarvi, dunque, le iniziative degli ultimi mesi ma, soprattutto, vorrei guardare avanti, dandovi una breve panoramica degli eventi futuri che ci vedranno all'opera nel 2014 con rinnovate speranze ed energie.

Dopo aver curato, infatti, la pubblicazione dei due Volumi dedicati al rene policistico, uno di carattere divulgativo, per i pazienti e i loro familiari, e uno di carattere più strettamente scientifico, rivolto ai medici, AIRP ha colto due delle più importanti occasioni nazionali per presentarli e distribuirli.

Stiamo parlando dell'evento di presentazione "Rene Policistico: Conoscerlo è già combatterlo!" che si è tenuto a Firenze il 27 Settembre in occasione del $54^{\circ}$ Congresso Nazionale SIN (Società Italiana di Nefrologia). Alla presenza del Presidente SIN Prof. Capasso e dei Professori Gesualdo, Scolari e Magistroni, l'evento ha visto la partecipazione di oltre quaranta persone, tra iscritti al Congresso e giornalisti.

La seconda presentazione dei Volumi si è tenuta ancora a Firenze il 21 Novembre, quando ho avuto l'opportunità di presentarli al $30^{\circ}$ Congresso Nazionale SIMG (Società Italiana di Medicina Generale), durante la cerimonia di inaugurazione, a una platea gremita di Medici di Medicina Generale. Ringrazio, quindi, il Dr. Claudio Cricelli, Presidente SIMG, per aver offerto ad AIRP questa grande opportunità.

Non dimentichiamo, infine, il $45^{\circ}$ Corso di aggiornamento in nefrologia e metodiche dialitiche della Fondazione D'Amico, in corso dal 5 all'8 Dicembre a Milano, un appuntamento annuale di grande rilievo, immancabile per la nostra Associazione, che, anche quest'anno, sarà presente con un desk presso lo spazio espositivo e proseguirà la distribuzione dei due Volumi dedicati al rene policistico. Come vi avevamo anticipato nel numero procedente, si sono svolti anche eventi regionali, non certo di minore importanza.

Per primo, in ordine temporale, il grande successo della " $2^{a}$ Tavola Rotonda AIRP con i Pazienti" e i loro familiari, a 


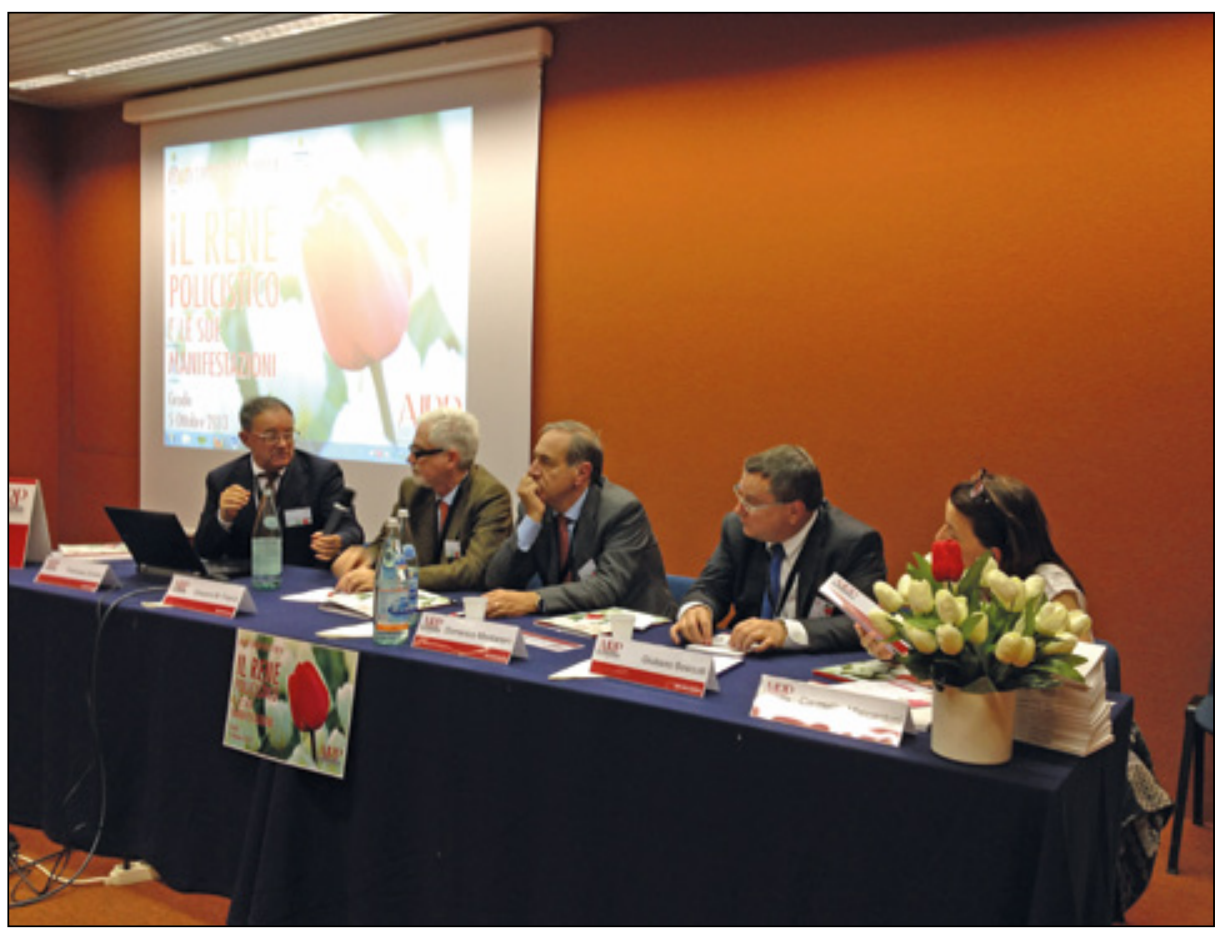

conclusione dei lavori del Congresso di Nefrologia "Genetica e Rene", che si è svolto il 5 Ottobre a Grado. La settimana successiva si è svolto il $13^{\circ}$ Convegno $A I R P$, un incontro di alto livello scientifico, volto a diffondere le conoscenze relative all'ADPKD e al trapianto di rene, organizzato il 12 Ottobre a Torino presso l'unità di Nefrologia, Dialisi e Trapianto dell'Ospedale Molinette.

Due momenti fondamentali di informazione e formazione che fanno parte del più ampio progetto di sensibilizzazione di AIRP e che hanno coinvolto alcuni tra i più illustri specialisti delle due Regioni.

Ed eccoci al 2014! È con grande piacere che vi annuncio il coinvolgimento di AIRP in un importante network internazionale dedicato alle malattie renali. In Gennaio, infatti, siamo stati invitati a partecipare al Congresso KDIGO, Kidney Disease: Improving Global Outcomes, di Edimbur- go, primo passo verso la definizione delle Linee Guida internazionali sul Rene Policistico Autosomico Dominante.

KDIGO è l'unica organizzazione mondiale a sviluppare e a implementare Linee Guida globali per le malattie renali. Questo appuntamento dà il via a un percorso molto importante per AIRP, che intraprenderemo insieme ai pazienti che ogni giorno ascoltiamo e rappresentiamo con l'obiettivo di prendere parte a un gruppo di lavoro internazionale e di dare voce alle istanze principali, ben oltre i confini nazionali.

Intanto, il Road Show prosegue con la prossima tappa: il 15 Febbraio 2014 a Bari, con la $14^{\mathrm{a}}$ tappa del Convegno "Il rene policistico e le sue manifestazioni”, presso l'Aula Magna della Facoltà di Medicina e Chirurgia-Ospedale AOU Policlinico Giovanni XXIII, organizzato grazie al prezioso contributo del Prof. Loreto Gesualdo e del Prof. Francesco Scolari.

I ringraziamenti per tutto quello che è stato possibile realizzare nel 2013 vanno a tutti voi, perché senza l'aiuto, le idee e il supporto di ciascuno non ce l'avremmo fatta. Nell'augurare a tutti le più serene festività natalizie, ricordo che, per affrontare il nuovo anno, avremo bisogno anche del vostro prezioso supporto e che è possibile sostenere AIRP con una donazione spontanea al $\mathrm{C} / \mathrm{C}$ postale 73690091 .

Un caro saluto e buona lettura! 